Journal of Animal and Veterinary Advances 10 (8): 1032-1036, 2011

ISSN: $1680-5593$

(C) Medwell Journals, 2011

\title{
Study on the Resistance of Extended Spectrum $\beta$-Lactamases
}

\author{
${ }^{1,2}$ Chun-Hui Zhang, ${ }^{2}$ Xiao-Gen Zhang, ${ }^{2}$ Yong-Shu Shen and ${ }^{1}$ Jian-Hua Wang \\ ${ }^{1}$ College of Animal Veterinary Medicine, \\ Northwest A and F University, Yangling, 712100 Shaanxi, P.R. China \\ ${ }^{2}$ Department of Pharmacy Engineering, \\ Zheng zhou College of Animal Husbandry Engineering, 450011 Henan, P.R. China
}

\begin{abstract}
The multi-drug resistance of bacteria is a serious threat to the treatment of clinical infectious diseases; Escherichia coli (E. coli) possess plasmid-encoded Extended Spectrum $\beta$-Lactamases (ESBLs) that confer multi-drug resistance. In order to overcome this kind of resistance, some experiments were done in this study. In this study for the clinic pathogenic E. coli, ESBLs genetype were detected with PCR and for the induced E. coli, the plasmid elimination effects of several plasmid-curing agents to ESBLs were investigated using SDS as a control. Results showed that the bacteria producing ESBLs were amplified TEM type ESBLs fully, two strains were amplified both TEM type ESBLs and SHV type ESBLs, only one strain was amplified both TEM type ESBLs and CTX type ESBLs. And to the induced E. coli C83845-y, the elimination rate of chlorpromazine, hydrochloride, diazepam, norfloxacin and ciprofloxacin was $14.53,13.50,14.59,8.43$ and $8.23 \%$, respectively, the elimination rate of SDS was $11.05 \%$, blank control was $0.05 \%$ to induce $E$. coli $\mathrm{C} 83907-\mathrm{y}$, the elimination rate of chlorpromazine, hydrochloride, diazepam, norfloxacin and ciprofloxacin was $11.25,12.23,13.51,9.91$ and $9.03 \%$, respectively, the elimination rate of SDS was $10.72 \%$, blank control was $0.05 \%$. These results indicated that ESBLs-positive $E$. coli in Henan have transmitted resistant gene plasmids among different bacteria, the tricyclic-psychotropic drugs were more effective at eliminating the ESBL-resistant plasmid. This study provides insight into strategies for overcoming the resistance conferred by ESBLs.
\end{abstract}

Key words: ESBLs, E. coli, multi-drug resistance, detection, elimination, China

\section{INTRODUCTION}

E. coli infection has become the primary infectious disease of chickens, resulting in $31,700,000$ deaths in China annually and causing large profit losses to the poultry industry (Dai et al., 2008). The $\beta$-lactam antibiotics as amoxicillin and ceftiofur had healed the diseases well before. But in recent years, the bacteria resistance reduced their effect seriously.

Strains of E. coli, producing ESBLs are the most important resistant mechanism to the third-generation cephalosporins and the new $\beta$-lactam-antibiotics (Davies et al., 2008). According to a previous report, the gene encoding ESBL is located on a plasmid and can be transmitted from one bacterial strain to another (Pitout and Laupland, 2008) so, bacteria producing ESBLs have multi-drug resistance consequently. When ESBLs-positive bacteria infection has been identified, there is a delay in initiating an appropriate antibiotic treatment (Akujobi et al., 2008). This resistance has attracted much attention (Bush, 2008; Canton et al., 2008). However, studis on ESBLs in veterinary clinic of Henan is quite rare. In addition to the using of $\beta$-lactamases inhibitors another method of overcoming ESBLsresistance was eliminating the resistant plasmid and reversing the resistance. Bacteria containing plasmids were cultured with normal medium and simultaneously eliminated by physical and chemical methods although, plasmid replication was suppressed but chromosome replication were still continuing so, the plasmid in offspring bacteria were eliminated. The elimination frequency by suppression is more than $10^{2}-10^{5}$ times the spontaneous elimination.

The methods of eliminate resistant plasmids which include physical methods, chemical methods, antibiotics and non-antibiotic methods (Kristiansen and Amaral, 1997). In this study in order to monitor the spread of ESBLs-resist plasmid, the ESBLs genetypes were detected. And in order to screen to the right plasmidcuring agents, the efficacy of several plasmid-curing agents against ESBLs in E. coli were evaluated too.

Corresponding Author: Jian-Hua Wang, College of Animal Veterinary Medicine, Northwest A and F University, Yangling, 712100 Shaanxi, P.R. China 


\section{MATERIALS AND METHODS}

Bacterial strains and culture conditions: The standard E. coli C83907 and C83845 were purchased from the China Institute of Veterinary Drug Control (Beijing, China). The 153 clinic strains of $E$. coli were isolated from distinct regions of Kaifeng, Xinzheng, Luohe, Luoyang, Nanyang and Xinxiang (Henan, China). Some strains were from Swine, the others were from chickens. All strains were cultured in Luria-Bertani (LB) medium at $37^{\circ} \mathrm{C}$.

Chemicals and enzymes: Gold view nucleic acid dye and D2000 were purchased from Beijing Solarbio Science and Technology (Beijing, China). Taq DNA polymerase and dNTP were purchased from Takara Biotechnology Co. Ltd. (Dalian, P.R. China). Cetiofur sodium, formulated as an intramuscular injection was purchased from Henan Pu-Like Co. Ltd (Henan, China).

Ciprofloxacin was purchased from Zhejiang Guobang Pharmaceutical Co., Ltd. (Zhejiang, China); the hydrochloric chlorpromazine and oxaprozin were purchased from Nantong No. 3 pharmaceutical factory (Jiangsu, China). The Sodium Dodecyl Sulfate (SDS) used as control in elimination experiments was purchased from Shanghai Shengzhong Fine Chemical Co. Ltd (Shanghai, China).

Detection of ESBLs: The 153 clinic bacteria isolates were detected by PCR. As described previously (Tenover et al., 1995) according to Plasmid Extraction Kit manual, the DNA was extracted. Quality of the extracted DNA was analyzed by electrophoresis on a $1.0 \%$ agarosegel and observed with UVI system (UVI tec ST John's Innovation Centre, English). The PCR primers for TEM: 5'-GAGTATTCAACATTTCCGTGTCGC-3' (forward) 5'-TACCAATGCTTAATCAGTGAGGC-3'(reverse),SHV: 5'-ATGCGTTATATTCGCCTGTG-3'(forward), 5'-TTAGCG TTGCCAGTGCTTGATC-3' (reverse) and CTX: 5'-GGGC TGAGATGGTGACAA AGAG-3' (forward), 5'-CGTGC GAGTTCGATTTATTCA AC- 3 ' (reverse).

The length of the expected amplified fragment was about 860 bp. The PCR amplification reactions were performed in a total volume of $50 \mu \mathrm{L}$. Each reaction mixture contained $2 \mu \mathrm{L}$ DNA templates, $1 \mu \mathrm{L}$ of each primer, $0.5 \mu \mathrm{L}$ of Taq DNA polymerase, $5 \mu \mathrm{L}$ of $10 \mathrm{x}$ buffer and $4 \mu \mathrm{L}$ of dNTP. PCR reaction was carried out in a Gene-Amp PCR system 2000 (Biometra, Gottinger, Germany).

PCR reaction program: The $\mathrm{PCR}$ program consisted of an initial denaturation step at $94^{\circ} \mathrm{C}$ for $10 \mathrm{~min}$ followed by 35 cycles consisting of denaturation at $94^{\circ} \mathrm{C}$ for $1 \mathrm{~min}$, annealing at $55^{\circ} \mathrm{C}$ for $1 \mathrm{~min}$ and primer extension $\mathrm{f}$ at $72^{\circ} \mathrm{C}$ or $1.5 \mathrm{~min}, 35$ cycles, the last extension step was extended to $8 \mathrm{~min}$ longer. PCR products were evaluated for the expected size and quantified on $1 \%$ agarosegels.

Induction of resistant bacteria producing ESBLs: To avoid the other resistant influence for the plasmid cure agents in the clinical resistant bacteria. In this study, the standard strains was induced by ceftiofur and the induced bacteria producing ESBLs was used the eliminated bacteria.

The standard bacteria was inoculated into medium containing the sub-inhibitory concentration drug and incubated for $16 \sim 18 \mathrm{~h}$ then frozen for $6 \sim 8 \mathrm{~h}$. After it was melted, the bacterium was inoculated into the medium containing higher concentration drug again. The above steps were repeated until bacteria had produced ESBLs.

Elimination of ESBL-resistant plasmid: After the MICs of plasmid-curing agents to the induced $E$. coli were detected, using the 2-fold dilution method as previously described (Nakamura et al., 2009) (Table 1). ESBLs resistant-plasmid elimination was carried out according to previous reports (Imre et al., 2006; Schelz et al., 2006). Firstly, the $E$. coli strains were inoculated onto LB agar plate for resuscitation then inoculated into $5 \mathrm{~mL}$ of $\mathrm{LB}$ broth and incubated shaking for $24 \mathrm{~h}$ at $37^{\circ} \mathrm{C}$. Secondly, $60 \mu \mathrm{L}$ of the germ culture containing $10 \mu \mathrm{L}$ plasmid-curing agents $(1 / 2 \mathrm{MIC})$ broth was prepared and incubated, shaking for $24 \mathrm{~h}$ at $37^{\circ} \mathrm{C}$. The above bacteria culture were then inculated onto 10-12 LB agar plates containing plasmid-curing agents, respectively and incubated for $24 \mathrm{~h}$ at $37^{\circ} \mathrm{C}$. Thirdly, $100-500$ single colonies were picked and inoculated onto LB agar plates containing ampicillin $\left(0.1 \mathrm{mg} \mathrm{mL}^{-1}\right)$ incubated for $24-48 \mathrm{~h}$ at $37^{\circ} \mathrm{C}$. Some colonies were inoculated onto agar plates without ampicillin as controls.

Table 1: MIC of plasmid-curing agents to $E$. coli

\begin{tabular}{lccccc}
\hline & C83907-y & C83845-y & C83907 & C83845 & C83907-x \\
Drug & $-\mathrm{C} 83907-\mathrm{x}$ & 74 & 74 \\
Chloropromazine & 74 & 74 & 74 & 74 & 74 \\
Hydrochloride & 74 & 74 & 74 & 74 & 74 \\
Diazepam & 74 & 32 & 0.1 & 0.1 & 0.1 \\
Norfloxacin & 16 & 16 & 0.05 & 0.05 & 0.05 \\
Ciprofloxacin & 8 & 74 & 0.05 \\
\hline
\end{tabular}

C83907-y was the induced bacteria using C83907, C83845-y was the induced bacteria using C83845. C83907-x was the elimated bacteria from C83907-y, C83845- $\mathrm{x}$ was the elimated bacteria from C83845-y 
Screening for expunction bacteria: After the $24 \sim 48 \mathrm{~h}$ incubation, bacteria did not grow on ampicillin-containing plates but grow on common plates were selected and subcultured again on ampicillin-containing plates. Bacteria did not grow after subculturing was classed as possible expunction bacteria.

Detection of expunction bacteria: Possible expunction bacteria were inoculated into $10 \mathrm{~mL}$ broth culture and incubated, shaking for $48 \mathrm{~h}$ at $37^{\circ} \mathrm{C}$. Plasmid DNA was extracted using a plasmid extraction kit (Takara Biotechnology Co. Ltd., Dalian, China), separated on 1.0\% agarose gels (Takara Biotechnology Co. Ltd.) and observed with a UVI system (UVI tec, ST John's Innovation Centre, UK) according to the manufacturer's instructions.

Calculation of the plasmid elimination rate: Bacteria which growed in ordinary plates but did not grow in plates containing antibiotic were chosed and confirmed in plates containing AP were recorded as bacteria eliminated ESBLs plasmid. Percentages of elimination were calculated.

The control experiment: The conventional plasmid eliminator agent SDS $(0.5 \%)$ was used as the control. Bacteria producing ESBLs were incubated in water at $45^{\circ} \mathrm{C}$, other steps are as same as the previous. Bacteria that had naturally eliminated the plasmid were used as a blank control.

\section{RESULTS AND DISCUSSION}

In 153 clinic strains, plasmids from ESBLs were extracted in $35 E$. coli. And 35 strains were amplified TEM type ESBLs fully, two strains were amplified TEM type ESBLs and SHV type ESBLs, only one strain was amplified TEM type ESBLs and CTX type ESBLs (Fig. 1-3) were pictures of these results.

PCR amplifications of extraction of ESBLs-resistant elimination before and after were showed in Fig. 4. It can be seen from the figure; ESBLs resistant-plasmid was clearly visible in the induced $E$. coli but not amplified in the eliminated bacteria. Elimination rate of agents on ESBL-resistant plasmid in vitro. All elimination results were showed in Table 2. For the induced C83845-y strain, the elimination rate of chlorpromazine, hydrochloride, diazepam, norfloxacin, ciprofloxacin was $14.53,13.50$, $14.59,8.43$ and $8.23 \%$, respectively, for the other induced bacteria (C83907-y), the elimination rate of chlorpromazine, hydrochloride, diazepam, norfloxacin, ciprofloxacin was $11.25,12.23,13.51,9.91$ and $9.03 \%$, respectively. And the elimination rates of SDS to C83845-y and C83907-y were 11.05 and $10.72 \%$, blank control was 0.05 and $0.06 \%$.

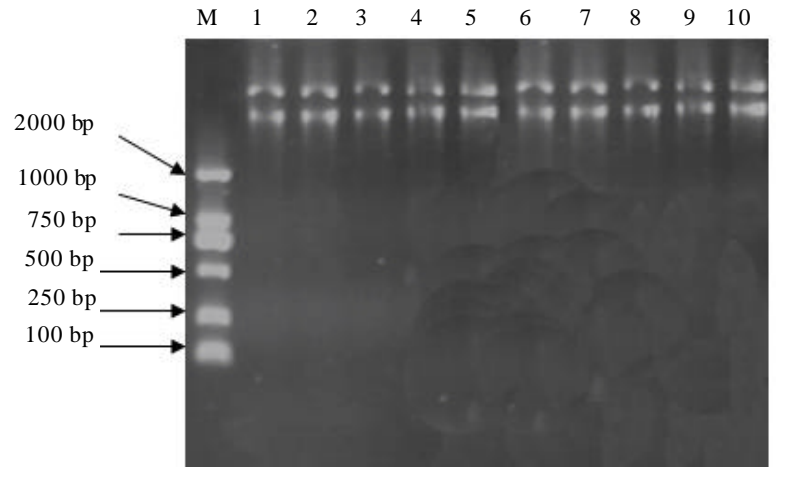

Fig. 1: Results of plasmid of some $E$. coli extracted of ESBLs M. Marker; 1 10 were E. coli in chickens from Kaifeng, Xinzheng, Luohe, Luoyang, Xingyang, Zhongmou and E. coli in Swine from Xinzheng, Xingyang, Anyang, Nanyang

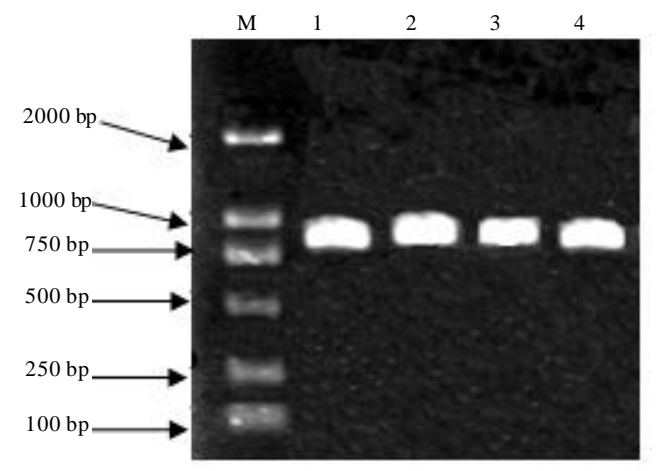

Fig. 2: PCR amplification of plasmid extracted from ESBIs M. Marker, 1, 2PCR amplification of TEM and SHV primer of ESBLs for $E$. coli from Swine in Anyang. 3,4 . PCR amplification of TEM and SHV primer of ESBLs for $E$. coli from Chicken in Xingyang

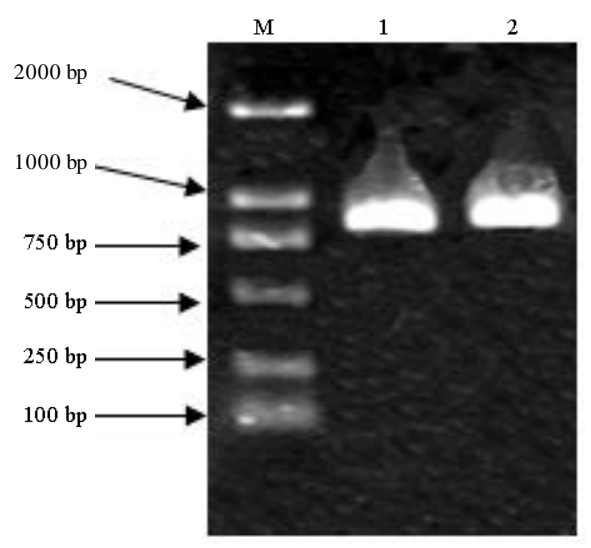

Fig. 3: PCR amplification of plasmid extracted from ESBIs M.Marker; 1,2 PCR amplification of TEM and CTX primer of ESBLs for $E$. coli from Chicken in Xinzheng 
Table 2: Elimination rate of plasmid-curing agents to plasmid of ESBLs in vitro

\begin{tabular}{|c|c|c|c|c|c|c|c|}
\hline \multirow[b]{2}{*}{ Bacteria } & \multicolumn{7}{|l|}{ Drug (\%) } \\
\hline & Chlorpromazine & Hydrochloride & Diazepam & Norfloxacin & Ciprofloxacin & SDS & Blank control \\
\hline C83845-y & 14.53 & 13.50 & 14.59 & 8.43 & 8.23 & 11.05 & 0.05 \\
\hline C83907-y & 11.25 & 12.23 & 13.51 & 9.91 & 9.03 & 10.72 & 0.06 \\
\hline
\end{tabular}

C83907-y was the induced bacteria using C83907, C83845-y was the induced bacteria using C83845

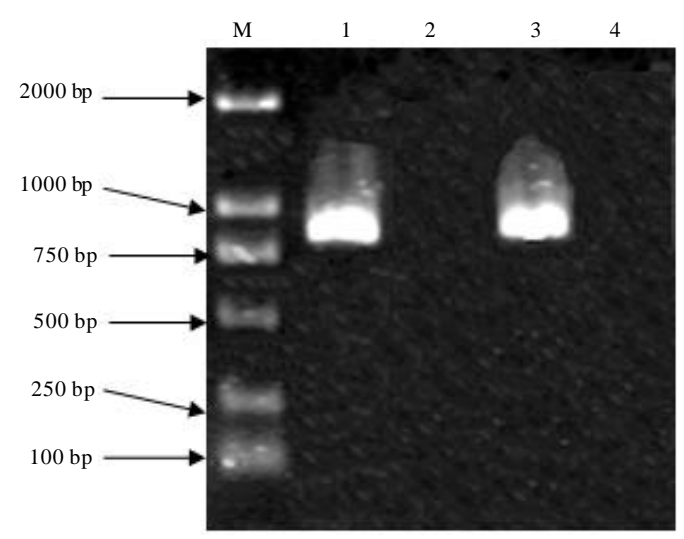

Fig. 4: PCR products of plasmid before/after elimination M. Marker; 1, 3. Before elimination of ESBLs in C83907-y and C83845-y; 2, 4. After elimination of ESBLs in C83907-y and C83845-y

Bacterial resistance is a public health problem worldwide. In recent years, bacterial resistance to $\beta$-lactam antibiotics has risen dramatically (Katayama et al., 2004). Contributing to this increase has been the spread of ESBLs. In this study, the producd bacteria ESBLs were amplified TEM type ESBLs fully. Itillustrated that TEM type ESBLs are the most ESBLs in veterinary clinice in Henan province, the $E$. coli from Anyang and Xingyang produced both TEM-type ESBLs and SHV-type ESBLs. $E$. coli from Xinzheng produced both TEM-type ESBLs and CTX-type ESBLs. This result illustrated that the clinic $E$. coli producing ESBLs have transmited resistant gene through plasmids in Henan.

Under antibiotic selection pressure, resistant plasmid bonded and transferred in the bacteria will increase the clinical therapeutic difficulty. Ceftiofur, a third-generation broad-spectrum cephalosporin has been used to treat diseases in Swine, ruminants and horses (Hornish and Kotarski, 2002). But after the standard strain were induced by it for 18 times in this study, it produced ESBLs and the MIC was increased significantly. This showed that the bacteria resistance was associated with the frequent use of drugs.

In the last 30 years, many researchers in the world have been actively looking for effective ways to eliminate resistant plasmids. In this study, the elimination rates of chlorpromazine, hydrochloride and diazepam were higher than natural elimination rate were similar with SDS. But the
SDS has higher toxicity was not suitable for using in vitro. And the MICs of tricyclic-psychotropic drugs to the standard strains C83845 and C83907 were same the MICs of them to the induced producing bacteria, this illustrated that tricyclic-psychotropic drugs have not pharmacology effect to $E$. coli, only played the elimination in the experiment. As reported by Molnar et al. (1992) and Kristiansen et al. (2007), the tricyclicpsychotropic drugs can eliminate hemolysin-encoded plasmid, increase the permeability of bacterial membrane and inhibit the activity of DNA helicase to format supercoiled plasmid, resulting in the inhabitation of plasmid replication. We can conclude that tricyclicpsychotropic drugs have the potential power to overcome the antibiotic resistance conferred by ESBLs.

\section{CONCLUSION}

These result shows that ESBLs-positive $E$. coli in Henan have not transmitted resistant gene through plasmids. The tricyclic-psychotropic drugs were proposed to be used as plasmid-cure agent in veterinary clinics in China.

\section{ACKNOWLEDGEMENTS}

The researchers thank Dr. Y.X. Yang for suggestions on manuscript preparation. This study was supported by the Key Science and Technology Program of Henan province, China (072102130009) and the Natural Science Fund of the Education Department of Henan Province, China (2006230004, 2009A230009).

\section{REFERENCES}

Akujobi, C.O., J.N. Ogbulie and C.S. Alisi, 2008. Occurrence of extended-spectrum â-lactamases in Escherichia coli isolated from piggery farms in Imo State, Nigeria. World J. Microbiol. Biotechnol., 24: $2167-2170$.

Bush, K., 2008. Extended-spectrum â-lactamases in North America, 1987-2006. Clin. Microbiol. Infect., 14: $134-143$.

Canton, R., A. Novais, A. Valverde, E. Machado, L. Peixe, F. Baquero and T.M. Coque, 2008. Prevalence and spread of extended-spectrum beta-lactamaseproducing Enterobacteriaceae in Europe. Clin. Microbiol. Infect., 14: 144-153. 
Dai, L., L.M. Lu, C.M. Wu, B.B. Li and S.Y. Huang et al., 2008. Characterization of antimicrobial resistance among Escherichia coli isolates from chickens in China between 2001 and 2006. FEMS Microbiol. Lett., 286: $178-183$.

Davies, T.A., W. Shang, K. Bush and R.K. Flamm, 2008. Activity of doripenem and comparator b-lactams against US clinical isolates of Streptococcus pneumoniae with defined mutations in the penicillinbinding domains of pbpla, pbp2b and pbp2x. J. Antimicrob. Chemother., 61: 751-753.

Hornish, R.E. and S.F. Kotarski, 2002. Cephalosporins in veterinary medicine-Ceftiofur use in food animals. Curr. Top. Med. Chem., 2: 717-731.

Imre, A., F. Olasz, J. Kiss and B. Nagy, 2006. A novel transposon-based method for elimination of large bacterial plasmids. Plasmid, 55: 235-241.

Katayama, Y., H.Z. Zhang and H.F. Chambers, 2004. PBP 2a mutations producing very-high-level resistance to â-lactams. Antimicrob. Agents Chemother., 48: 453-459.

Kristiansen, J.E. and L. Amaral, 1997. The potential of resistant infection with non-antibiotics. J. Antimicrob. Chemother., 40: 319-327.
Kristiansen1, J.E., O. Hendricks, T. Delvin, T.S. Butterworth and L. Aagaard et al., 2007. Reversal of resistance in microorganisms by help of nonantibiotics. J. Antimicrob. Chemother., 59: 1271-1279.

Molnar, J., S. Foldeak, M.J. Nakamura, H. Rausch, K. Domonkos and M. Szabo, 1992. Antiplasmid activity: Loss of bacterial resistance to antibiotics. Acta Pathol. Microbiol. Immunol. Scand., 30: 24-31.

Nakamura, T., C. Shimizu, M. Kasahara, K. Okuda and C. Nakata et al., 2009. Monte Carlo simulation for evaluation of the efficacy of carbapenems and new quinolones against ESBL-producing Escherichia coli. J. Infect. Chemother., 15: 13-17.

Pitout, J.D.D. and K.B. Laupland, 2008. Extendedspectrum â-lactamase-producing enterobacteriaceae: An emerging public-health concern. Lancet Infect. Dis., 8: 159-166.

Schelz, Z., J. Molnar and J. Hohmann, 2006. Antimicrobial and antiplasmid activities of essential oils. Fitoterapia, 77: 279-285.

Tenover, F.C., R.D. Arbeit, R.V. Goering, P.A. Mickelsen, B.E. Murray, D.H. Persing and B. Swaminathan, 1995. Interpreting chromosomal DNA restriction patterns produced by pulsed-field gel electrophoresis: Criteria for bacterial strain typing. Clin. Microbiol., 33: 2233-2239. 